\title{
Extended Kalman Filter Channel Estimation for Line-of-Sight Detection in WCDMA Mobile Positioning
}

\author{
Abdelmonaem Lakhzouri \\ Institute of Communications Engineering, Tampere University of Technology, P.O. Box 553, 33101 Tampere, Finland \\ Email: abdelmonaem.lakhzouri@tut.fi \\ Elena Simona Lohan \\ Institute of Communications Engineering, Tampere University of Technology, P.O. Box 553, 33101 Tampere, Finland \\ Email: elena-simona.lohan@tut.fi
}

Ridha Hamila

Etisalat College of Engineering, Emirates Telecommunications Corporation, P.O. Box 980, Sharjah, UAE Email: hamila@ece.ac.ae

Markku Renfors

Institute of Communications Engineering, Tampere University of Technology, P.O. Box 553, 33101 Tampere, Finland Email: markku.renfors@tut.fi

Received 21 October 2002 and in revised form 29 May 2003

\begin{abstract}
In mobile positioning, it is very important to estimate correctly the delay between the transmitter and the receiver. When the receiver is in line-of-sight (LOS) condition with the transmitter, the computation of the mobile position in two dimensions becomes straightforward. In this paper, the problem of LOS detection in WCDMA for mobile positioning is considered, together with joint estimation of the delays and channel coefficients. These are very challenging topics in multipath fading channels because LOS component is not always present, and when it is present, it might be severely affected by interfering paths spaced at less than one chip distance (closely spaced paths). The extended Kalman filter (EKF) is used to estimate jointly the delays and complex channel coefficients. The decision whether the LOS component is present or not is based on statistical tests to determine the distribution of the channel coefficient corresponding to the first path. The statistical test-based techniques are practical, simple, and of low computation complexity, which is suitable for WCDMA receivers. These techniques can provide an accurate decision whether LOS component is present or not.
\end{abstract}

Keywords and phrases: extended Kalman filter, fading statistics, LOS detection, mobile positioning, WCDMA systems.

\section{INTRODUCTION}

For the public interest, mobile phone positioning in a cellular network with reliable and rather accurate position information has become unavoidable after the Federal Communications Commission mandate, FCC-E911 docket on emergency call positioning in USA, and the coming E112 in the European Union [1]. One method for locating the mobile station (MS) in two dimensions requires the measurement of line-of-sight (LOS) distance between the MS and at least three base stations (BSs). Hence, knowing which BS is reporting, LOS component is crucial for accurate position estimation. In many cases, the non-LOS (NLOS) signal compo- nents, arriving with delay less than one chip at the receiver, obscure the LOS signal. This situation of overlapping multipath propagation is one of the main sources of mobile positioning errors $[2,3,4]$.

Previous studies dealing with LOS detection used range measurement-based techniques $[5,6,7]$ (i.e., measurements of the time of arrival), which exploit the time history of the range measurements and the a priori knowledge of the noise floor in the system. These techniques can increase the accuracy of the mobile position estimation, but they require the knowledge of the a priori statistic parameters such as the standard deviation of the measurement noise. The use of a link level-based techniques where the signal processing 
is made in the MS side as presented in this paper to detect whether the LOS component is present or not is a new topic. In this paper, accurate estimates of the channel coefficients and their corresponding delays in the context of closely spaced paths are obtained using extended Kalman filter (EKF) algorithm, aided by an interference cancellation (IC) technique. The channel coefficients will be used as basis for deciding whether the first arriving path is a LOS or NLOS component.

Many techniques were presented to cope with closely spaced multipath propagations, such as subspace-based methods [8] or least square (LS) approaches $[9,10]$. These techniques can provide rather accurate estimation of the multipath delays, but they suffer from the high complexity for the implementation in WCDMA systems in tracking mode. Few authors have studied the problem of joint parameters estimation using Kalman filtering in multipath fading and multiuser environment. In [11], Iltis has developed a new technique for jointly estimating the channel coefficients and the first-path delay in frequency selective channel based on Kalman filtering in a single user system. Recently, the idea has been extended to multiuser scenario [12]. In order to solve the closely spaced multipaths, we propose here an EKF-based solution with IC scheme. EKF algorithm jointly estimates the delays and complex coefficients of all the paths from all the participating BSs and it is combined with a new IC scheme to enhance the estimation of the channel from the desired BS (serving BS). The obtained estimates are used to detect whether the LOS component is present or not. The detection procedure exploits the distribution of the first arriving path. If the distribution is Rician with strong Rician factor, then LOS component is likely to be present. If the distribution is Rayleigh, it is more likely that LOS component is absent. We point out that the proposed algorithm is not limited to a WCDMA system and it can be easily extended to other mobile positioning systems.

This paper is organized as follows. In Section 2, the channel and signal model are described. Then, the joint estimation of the channel coefficients and delays is described in Section 3 with an emphasis on the proposed IC algorithm. Section 4 is devoted to the novel LOS detection procedures. Simulation results are provided in Section 5, and conclusions are drawn in Section 6.

\section{CHANNEL AND SIGNAL MODELS}

The system under consideration is a DS-CDMA system with $N_{\text {BS }}$ base stations and $N_{u}$ users per BS. In baseband system (fully digital implementation), the received signal complex valued at sample level, transmitted over an $L$-path fading channel, can be written as [13]

$$
r(i)=\sum_{m=-\infty}^{\infty} \sum_{u=1}^{N_{\mathrm{BS}}} \sum_{l=1}^{L} \sqrt{E_{b_{u}}} \alpha_{l, u}(m) s_{u}^{(m)}\left(i T_{s}-\tau_{l, u}(m)\right)+\eta(i)
$$

where $i$ is the sample index (we assume that there are $N_{s}$ samples per chip), $E_{b_{u}}$ is the bit energy of the $u$ th BS (we assume that all bits of the same BS have the same energy), $L$ is the number of discrete multipath components, $T_{s}$ is the sampling period ( $T_{s}=T_{c} / N_{s}, T_{c}$ is the chip period), $\alpha_{l, u}(m)$ and $\tau_{l, u}(m)$ represent, respectively, the complex-valued time varying channel coefficient and delay of the $l$ th path of base station $u$, during the $m$ th symbol. The delays are treated as complex values, but only the magnitudes rounded to the nearest integer values are retained. We denote by $s_{\mathcal{u}}^{(m)}(\cdot)$ the signature of the $u$ th BS during symbol $m$ including data modulation, spreading code, and pulse shaping, and $\eta$ is an additive circular white Gaussian noise of zero mean and double-sided spectral power density $N_{0}$. The signatures of all users are assumed to be known at the receiver (this corresponds to a situation when a pilot signal is available, e.g., Common Pilot Channel (CPICH) signal in downlink WCDMA environment [14]). The intracell interference is assumed Gaussian distributed by virtue of central limit theorem, and it is included in the term $\eta(\cdot)$.

The output of the matched filter corresponding to the desired BS $u$ during the symbol $n$ with lag $\tau$ is as follows:

$$
y_{u}(n, \tau)=\sum_{v=1}^{N_{\mathrm{BS}}} \sum_{l=1}^{L} \sqrt{E_{b_{v}}} \alpha_{l, v}(n) \mathscr{R}_{u, v}\left(\tau-\tau_{l, v}(n)\right)+\tilde{\eta}(n),
$$

where $\mathscr{R}_{u, v}(\cdot)$ is the cross correlation between the signature of the BS of interest ( $u$ th BS) and the signature of the $v$ th BS, $\tilde{\eta}(n)$ is the filtered noise plus interchip and intersymbol interference, and $\alpha_{l, v}(n)$ and $\tau_{l, v}(n)$ are the complex channel coefficients and the path delays, respectively, at symbol level. We point out that the channel coefficients and delays are assumed to be constant within one symbol. This assumption is reasonable since the symbol period (e.g., $66.5 \mu \mathrm{s}$ for $S_{F}=256$ ) is much less than the coherence time of the channel. The constant delays assumption is also reasonable for terrestrial communications due to the negligible Doppler shift. The channel coefficients and delays are modeled as a Gauss-Markov process [11, 12, 15]

$$
\begin{aligned}
& \alpha_{l, v}(n+1)=\beta_{v} \alpha_{l, v}(n)+w_{\alpha_{l, v}}(n), \\
& \tau_{l, v}(n+1)=\gamma \tau_{l, v}(n)+w_{\tau_{l, v}}(n),
\end{aligned}
$$

where $w_{\alpha}$ and $w_{\tau}$ are mutually independent additive circular white Gaussian noise processes, $\gamma$ is a coefficient accounting for the delay variation, and $\beta_{v}$ is a coefficient accounting for the maximum Doppler spread, $f_{D}$, of the $v$ th BS, defined as [16]

$$
\beta_{v}=I_{0}\left(2 \pi f_{D} T_{\text {sym }}\right),
$$

where $I_{0}(\cdot)$ is the zero-order Bessel function and $T_{\text {sym }}$ is the symbol interval. We assumed that for each BS, all the paths have the same maximum Doppler spread. The coefficient $\beta_{v}$ is close to unity when the Doppler spread is significantly less than the Nyquist bandwidth. We assume here that the coefficient $\gamma$ is constant for all the BSs and all the paths. This is a reasonable assumption in terrestrial communication when the Doppler shift is negligible, and $\gamma$ can be set to a value close to unity for all multipath delays of all users. However, 
EKF can be easily modified to use different $\gamma$ coefficients [12]. We point out that the channel models of $[11,12,17]$ are different from (3) in the sense that, earlier, the paths have been assumed uniformly spaced at chip period $\left(T_{c}\right)$, and the only delay modeled with (3) is the delay of the first path. In this paper, we derive an extension of the EKF model for all the path delays. This should not affect the EKF algorithm; it will only increase slightly the number of parameters to be estimated, and hence, the complexity. Also, we point out that the Gaussian assumption of multiple access interference (MAI) can be relaxed and the algorithm is straightforward to extended to non-Gaussian MAI case by using some IC within each cell in a similar manner to the intercell IC algorithm presented in the next section.

\section{JOINT CHANNEL COEFFICIENTS AND PATH DELAYS ESTIMATION}

The joint estimation of multipath delays and complex channel coefficients of the serving BS is done in two steps. First, we jointly estimate all the path delays and channel coefficients from all participating BSs, which leads to an estimation of the interference due to CPICH channels. Then, an IC scheme will be combined to enhance the estimation of the desired BS (serving BS) channel. During the first step, the discrete state vector, $\mathbf{x}(n) \in \mathbb{C}^{2 L N_{\mathrm{BS}} \times 1}$, associated with all BSs is defined by

$$
\mathbf{x}(n)=\left[\mathbf{x}_{1}, \ldots, \mathbf{x}_{N_{\mathrm{BS}}}\right]^{T},
$$

where $\mathbf{x}_{v}=\left[\alpha_{1, v}(n), \ldots, \alpha_{L, v}(n), \tau_{1, v}(n), \ldots, \tau_{L, v}(n)\right]$, for $v=$ $1, \ldots, N_{\mathrm{BS}}$. Due to the fact that the received signal is not a linear function of the multipath delays $\tau_{l, v}$, an EKF is needed. The state and observation models are described by the following equations, respectively,

$$
\begin{gathered}
\mathbf{x}(n+1)=\mathbf{F} \mathbf{x}(n)+\mathbf{w}(n), \\
\mathbf{z}(n)=\mathscr{H}(\mathbf{x}(n))+v(n),
\end{gathered}
$$

where $\mathbf{w}(\cdot)$ and $\nu(\cdot)$ are circular white Gaussian noise processes, $\mathbf{F} \in \mathbb{R}^{2 L N_{\mathrm{BS}} \times 2 L N_{\mathrm{BS}}}$ is defined by $\mathbf{F}=\operatorname{Block} \operatorname{diag}\left(F_{1}, \ldots\right.$, $\left.F_{N_{\mathrm{BS}}}\right)$, where $F_{v}=\operatorname{diag}(\beta, \ldots, \beta, \gamma, \ldots, \gamma), \mathbf{z}(n)$ is the observation vector which depends nonlinearly on the state vector $\mathbf{x}(n), \mathbf{z}(n)=\left[y_{1}(n), \ldots, y_{N_{\mathrm{BS}}}(n)\right]^{T}$, and the nonlinear transform $\mathscr{H}(\cdot)$ is given as follows:

$$
\mathscr{H}(\mathbf{x}(n))=\left[H_{1}(\mathbf{x}(n)), \ldots, H_{N_{\mathrm{BS}}}(\mathbf{x}(n))\right]^{T},
$$

where $H_{i}(\mathbf{x}(n))=\sum_{v=1}^{N_{\mathrm{BS}}} \sum_{l=1}^{L} \sqrt{E_{b_{v}}} \alpha_{l, v}(n) \mathscr{R}_{i, v}\left(n T_{\mathrm{sym}}-\tau_{l, v}(n)\right)$, for $i=1, \ldots, N_{\mathrm{BS}}$.

Here, we assume that we have no data modulation, which is true for the CPICH reference channels used for positioning in WCDMA [14]. However, this assumption is not crucial in the sense that data can be removed in a decision-directed mode before we proceed with EKF estimation. The circular white Gaussian noise vector $\mathbf{w}(\cdot)$ is defined as

$$
\mathbf{w}(n)=\left[\mathbf{w}_{1}(n), \ldots, \mathbf{w}_{N_{\mathrm{BS}}}(n)\right]^{T},
$$

where $\mathbf{w}_{i}(n)=\left[w_{\alpha_{0, i}}, \ldots, w_{\alpha_{L-1, i}}, w_{\tau_{0, i}}, \ldots, w_{\tau_{L-1, i}}\right]$.
The EKF algorithm requires the linearization of the transform $\mathscr{H}(\cdot)$. The most common linearization method used is the first-order Taylor expansion defined as follows $[11,17,18]$ :

$$
\begin{aligned}
\mathscr{H}(\mathbf{x}(n)) \approx & \mathscr{H}(\hat{\mathbf{x}}(n \mid n-1)) \\
& +\sum_{m=1}^{2 L N_{\mathrm{BS}}}\left(\mathbf{x}_{m}(n)-\hat{\mathbf{x}}_{m}(n \mid n-1)\right) \\
& \times\left.\frac{\partial}{\partial \mathbf{x}_{m}} \mathscr{H}(\mathbf{x}(n))\right|_{\mathbf{x}(n)=\hat{\mathbf{x}}(n \mid n-1)},
\end{aligned}
$$

where $\hat{\mathbf{x}}(n \mid n-1)$ is the predictor at step $n$ conditional to previous observations, $\mathbf{x}_{m}(n)$ are the elements of the state vector $\mathbf{x}(n)$, and $\hat{\mathbf{x}}_{m}(n \mid n-1)$ are the elements of the predictor vector $\hat{\mathbf{x}}(n \mid n-1), m=1, \ldots, 2 L N_{\mathrm{BS}}$.

Using the linearization in (9), the set of EKF equations can be written as $[11,12,17]$

$$
\begin{aligned}
\hat{\mathbf{x}}(n \mid n) & =\hat{\mathbf{x}}(n \mid n-1)+\mathbf{K}(n)[\mathbf{z}(n)-\mathscr{H}(\hat{\mathbf{x}}(n \mid n-1))], \\
\mathbf{K}(n) & =\mathbf{P}(n \mid n-1) \mathscr{H}^{\prime}(n)\left[\mathscr{H}^{\prime}(n)^{H} \mathbf{P}(n \mid n-1) \mathscr{H}^{\prime}(n)+\Sigma_{\nu}\right]^{-1}, \\
\mathbf{P}(n \mid n) & =\left[\mathbf{I}-\mathbf{K}(n) \mathscr{H}^{\prime}(n)^{H}\right] \mathbf{P}(n \mid n-1) .
\end{aligned}
$$

Here, $\Sigma_{v}$ is the covariance matrix of the measurement noise and $\mathcal{H}^{\prime}(n)$ is the partial derivative matrix

$$
\mathscr{H}^{\prime}(n)=\left[\mathscr{H}_{1}^{\prime}, \ldots, \mathscr{H}_{N_{\mathrm{BS}}}^{\prime}\right]
$$

where

$$
\begin{aligned}
\mathscr{H}_{i}^{\prime}= & {\left[\frac{\partial \mathcal{H}(\hat{x}(n \mid n-1))}{\partial \hat{x}_{1, i}}, \ldots, \frac{\partial \mathcal{H}(\hat{x}(n \mid n-1))}{\partial \hat{x}_{L, i}},\right.} \\
& \left.\frac{\partial \mathscr{H}(\hat{x}(n \mid n-1))}{\partial \hat{x}_{L+1, i}}, \ldots, \frac{\partial \mathcal{H}(\hat{x}(n \mid n-1))}{\partial \hat{x}_{2 L, i}}\right]^{H} .
\end{aligned}
$$

To ensure real and integer values for the estimated delays, $\hat{x}_{j, i}(\cdot)$ are the rounded to the nearest integer value of $\left|\tau_{j, i}(\cdot)\right|$ for $j=L+1, \ldots, 2 L$, and for $i=1, \ldots, N_{\mathrm{BS}}$. The one step predictions of the state vector and error covariance matrix satisfy, respectively,

$$
\begin{aligned}
\hat{\mathbf{x}}(n+1 \mid n) & =\mathbf{F} \hat{\mathbf{x}}(n \mid n), \\
\hat{\mathbf{P}}(n+1 \mid n) & =\mathbf{F} \hat{\mathbf{P}}(n \mid n) \mathbf{F}^{T}+\mathbf{Q},
\end{aligned}
$$

where $\mathbf{Q}=\operatorname{Block} \operatorname{diag}\left(Q_{1}, \ldots, Q_{N_{\mathrm{BS}}}\right)$ and

$$
Q_{i}=\operatorname{diag}\left(\sigma_{w_{\alpha_{0, i}}}^{2}, \ldots, \sigma_{w_{\alpha_{L-1, i}}}^{2}, \sigma_{w_{\tau_{0, i}}}^{2}, \ldots, \sigma_{w_{\tau_{L-1, i}}}^{2}\right) .
$$

When the first stage of estimating all the path delays and channel coefficients is achieved, it becomes possible to estimate the interference $\hat{y}_{\text {int }}(n, \tau)$ coming from the nonserving BSs (we suppose that the serving BS has the index 1):

$$
\hat{y}_{\text {int }}(n, \tau)=\sum_{v=2}^{N_{\mathrm{BS}}} \sum_{l=1}^{L} \sqrt{E_{b_{v}}} \hat{\alpha}_{l, v}(n) \mathscr{R}_{1, v}\left(\tau-\hat{\tau}_{l, v}(n)\right) .
$$

To refine the estimation of the desired BS channel, we cancel 
the estimated interference

$$
\hat{y}_{\text {des }}(n, \tau)=y_{1}(n, \tau)-\hat{y}_{\text {int }}(n, \tau)
$$

and, then, we introduce a second estimation stage based on EKF with a state vector $\mathbf{x}^{\operatorname{des}}(n) \in \mathbb{C}^{2 L \times 1}$ and with an observation vector $\mathbf{z}^{\text {des }}(n)$ given, respectively, by

$$
\begin{aligned}
& \mathbf{x}^{\operatorname{des}}(n)=\left[\alpha_{1,1}(n), \ldots, \alpha_{L, 1}(n), \tau_{1,1}(n), \ldots, \tau_{L, 1}(n)\right]^{T}, \\
& \mathbf{z}^{\operatorname{des}}(n)=\hat{y}_{\text {des }}(n) .
\end{aligned}
$$

The EKF set of equations for single BS channel estimation can be retrieved easily from the equation presented for multiple BSs case. In this algorithm, we try to cancel only the interference coming from other BSs (interference due to $\mathrm{CPICH}$ channels). The interference coming from the other users (i.e., DPCH channels [14]) is considered as additive white noise and it will be neglected by the IC algorithm for simplicity. To cancel the intracell interference, the spreading codes of all users should be known by the receiver. Besides, in WCDMA systems, CPICH power is usually significantly higher than the individual DPCH power [14]. Therefore, using only intercell interference in the interference canceller is reasonable.

\section{LOS DETECTION}

The probability density function (pdf) of a fading channel with amplitude $|\alpha|$ which relates the Rayleigh, Rician, and Nakagami distributions is given by [19]

$$
\begin{aligned}
p_{r}\left(|\alpha|, \Omega, K_{r}\right)= & \frac{2|\alpha|\left(1+K_{r}\right)}{\Omega} \exp \left(-K_{r}-\frac{|\alpha|^{2}\left(1+K_{r}\right)}{\Omega}\right) I_{0} \\
& \times\left(2|\alpha| \sqrt{\frac{K_{r}\left(1+K_{r}\right)}{\Omega}}\right),
\end{aligned}
$$

where $\Omega$ is the average fading power, $\Omega=E\left[|\alpha|^{2}\right]$, and $K_{r}$ is the Rician factor. For $K_{r}=0$, the pdf becomes Rayleigh distribution and it is Nakagami- $n$ when $n^{2}=K_{r}$. We point out here that the Rayleigh distribution is a particular case of Nakagami and Rician for $n^{2}=K_{r}=0$. The question is how to detect the LOS and NLOS situations. This detection problem can be redefined in terms of a statistical test. First, we estimate $\left\{\left(\alpha_{i, 1}, \tau_{i, 1}\right), i=1, \ldots, N_{\mathrm{BS}}\right\}$ with the EKF algorithm. Then, by using statistic tests, we check if the channel is Rayleigh or not.

The most straightforward method is to estimate the pdf of the first arriving path, and compare it to some reference pdfs such as Rayleigh, Rician, Normal, Lognormal. To estimate correctly the distribution of the first arriving path, a set of independent fading coefficients are needed. The fading coefficients can be considered independent if they are at least a coherence time $\left(\Delta t_{\text {coh }}\right)$ apart. When the carrier frequency is $2.15 \mathrm{GHz}$, and for a mobile velocity $v$ in $\mathrm{m} / \mathrm{s}$, the coherence time is [20]

$$
\Delta t_{\mathrm{coh}}=\frac{9}{16 \pi f_{D}} \simeq \frac{0.025}{v}
$$

In WCDMA mobile positioning, two techniques have been proposed to let the MSs measure different BSs within their coverage. The first one is the idle period-downlink (IP-DL) transmission proposed in [21]. It imposes to each BS to turn off its transmission for a well-defined period of time to let the MSs measure other BSs. In this case, the MS cannot measure continuously all the links, and the number of independent points sufficient for the positioning can be only acquired from the serving BS. As an alternative to IP-DL method, Jeong et al. [22] proposed an IC scheme in conjunction with the delay lock loops (DLLs) to reduce the intercell interference. By using this technique, the MS can measure continuously all the BSs in its coverage. In our algorithm, we use the EKF-based IC scheme to be able to measure continuously all available links.

We consider that $N$ independent values are available in the MS memory to be used in the estimation of the channel distribution whenever the positioning is needed. For these $N$ independent points $x_{i}$, we test the hypothesis that $P_{d f}=Q_{d f}$, where $P_{d f}$ is the measured pdf and $Q_{d f}$ is the reference pdf (e.g., Rayleigh, Rician, etc.). We define the two states $H_{0}$ and $H_{1}$, respectively, such that [23]

$$
\begin{array}{ll}
P_{d f}\left(x_{i}\right)=Q_{d f}\left(x_{i}\right) & \text { for } 1 \leq i \leq N \\
P_{d f}\left(x_{i}\right) \neq Q_{d f}\left(x_{i}\right) & \text { for some } i .
\end{array}
$$

We introduce the $m$ events $X_{i}=\left\{x_{i-1}<x \leq x_{i}\right\}, i=$ $1, \ldots, m$, where $x_{0}=-\infty$ and $x_{m}=+\infty$. We denote by $k_{i}$ the number of successes of $X_{i}$, that is, the number of samples in the interval $\left[x_{i-1}, x_{i}\right]$.

Under the hypothesis $H_{0}$,

$$
\begin{gathered}
P\left(X_{i}\right)=P_{d f}\left(x_{i}\right)=Q_{d f}\left(x_{i}\right), \\
p_{i 0}=\left(x_{i}-x_{i-1}\right) P\left(X_{i}\right) .
\end{gathered}
$$

Thus, to test the hypothesis, we form the Pearson's test statistic (PTS) [23]

$$
\mathrm{PTS}=\sum_{i=1}^{m} \frac{\left(k_{i}-n p_{i 0}\right)^{2}}{n p_{i 0}},
$$

where $n$ is the total number of observed samples $(n \cong$ $\left.N \Delta t_{\text {coh }}\right)$. The hypothesis $H_{0}$ is accepted if the PTS value satisfies PTS $<\chi_{1-\lambda}^{2}(m-1)$, where $\chi_{1-\lambda}^{2}(m-1)$ is taken from the standard chi-square tables corresponding to the confidence level $\lambda$ and to the degree of freedom $(m-1)$.

This technique is efficient when the observation interval is long enough, the simulation results showed that around 1 second is needed to make reliable decision for a mobile velocity of $22.22 \mathrm{~m} / \mathrm{s}$. To decrease the duration of the observation and hence the hardware needed for storage, we propose a new algorithm using the estimation of Rician factor parameter $K_{r}^{v}$ with respect to the channel profile of the $v$ th BS defined by [20]

$$
K_{r}^{v}=\frac{\mu^{2}}{2 \sigma^{2}},
$$


For $i=1, \ldots, N_{\mathrm{BS}}$

Compute $K_{r}^{(i)}(\mathrm{dB})$

Evaluate $P_{\mathrm{NLOS}}^{(i)}$ and $P_{\mathrm{LOS}}^{(i)}$

Evaluate $d_{i}=P_{\mathrm{LOS}}^{(i)}-P_{\mathrm{NLOS}}^{(i)}$

if $d_{i}>0$, then LOS component is present from $\mathrm{BS}_{i}$ with probability $P_{\mathrm{LOS}}^{(i)}$.

else, LOS component is absent from $\mathrm{BS}_{i}$ with probability $P_{\mathrm{NLOS}}^{(i)}$. Next BS.

Algorithm 1: Rician factor-based LOS detection.

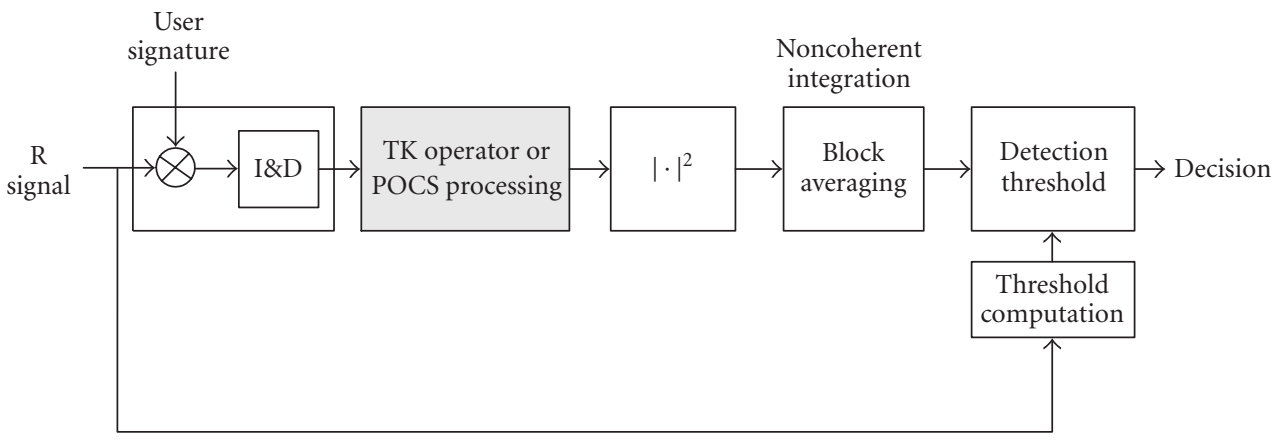

FIGURE 1: Block diagram of the acquisition model.

where $\mu=\left|E\left[\alpha_{1, v}\right]\right|$ and $\sigma^{2}=\operatorname{Var}\left[\alpha_{1, v}\right] / 2$. Hereinafter, we consider the case of single BS and the subscript $v$ will be dropped for convenience. In multiple BSs case, the same procedure is repeated for each BS. We point out that when $K_{r}$ is zero, $\mu$ is also zero and Rayleigh distribution should be detected. To distinguish between Rayleigh and Rician cases, we divide the whole range of $K_{r}$, in $\mathrm{dB}$ scale, into three regions: region I: $\left[-\infty, B_{\min }\right]$, region II: $\left[B_{\min }, B_{\max }\right]$, and region III: $\left[B_{\max },+\infty\right]$, where $B_{\min }$ and $B_{\max }$ are two predefined parameters, which depend on the level of noise in the system.

If $K_{r}(\mathrm{~dB}) \in$ region I, then the distribution is Rayleigh and we set the probability $\left(P_{\mathrm{NLOS}}, P_{\mathrm{LOS}}\right)$ to $(1.0,0.0)$, if $K_{r}(\mathrm{~dB}) \in$ region III, then the distribution is Rician and we set the probability $\left(P_{\mathrm{NLOS}}, P_{\mathrm{LOS}}\right)$ to $(0.0,1.0)$, and if $K_{r}(\mathrm{~dB}) \in$ region II, then the probabilities $P_{\mathrm{NLOS}}$ and $P_{\mathrm{LOS}}$ are computed as follow.

The range $\left[B_{\min }, B_{\max }\right]$ is divided into $(M+1)$ equally spaced intervals $\left[b_{i-1}, b_{i}\right]$, where $b_{0}=B_{\min }$ and $b_{M+1}=$ $B_{\max }$. If $b_{i-1} \leq K_{r}(\mathrm{~dB}) \leq b_{i}$, then we set the probability $\left(P_{\mathrm{NLOS}}, P_{\mathrm{LOS}}\right)$ to $((M-i+1) / M,(i-1) / M)$. This technique is simple to implement and provides accurate detection of the LOS component. The simulation showed that around 10 milliseconds are needed to detect accurately the distribution of the first arriving path. The algorithm for LOS detection based on the measurement from all BSs is shown in Algorithm 1.

\section{SIMULATION RESULTS}

The EKF-based estimation was simulated in tracking mode. We assume that the initial multipath delay estimates are within $N_{\text {init }}$ samples away from the true delays, where $N_{\text {init }} \leq$ $N_{s}$. The acquisition of the closely spaced multipath delays can be done with a separate feed-forward acquisition based on correlation and additional signal processing such as the nonlinear Teager Kaiser (TK) operator-based estimation [24], the iterative LS-based algorithms, projection onto convex sets (POCS) $[9,10,25]$, or the pulse subtraction (PS)-based algorithms $[26,27]$. The simulation results showed that the most promising algorithms are TK and POCS. Figure 1 shows the block diagram of the acquisition model including the additional signal processing.

The discrete-time TK operator applied to a complex signal $x(n)$ is given by $[27,28]$

$$
\begin{aligned}
\Psi_{d}(x(n))= & x(n-1) x(n-1)^{*} \\
& -0.5\left[x(n-2) x(n)^{*}+x(n) x(n-2)^{*}\right] .
\end{aligned}
$$

TK exploits the structure of the cross-correlation function to estimate the subchip-spaced multipath components [24, 28].

The POCS algorithm is a constrained deconvolution approach, originally proposed in $[9,25]$ for delay estimation in Rake receivers, under the assumption of rectangular pulse shapes. If we reformulate (2) into a vectorial form, it is possible to write the following expression:

$$
\mathbf{y}_{u}(n)=\mathbf{G}_{u, u} \mathbf{h}_{u}(n)+\mathbf{v}_{\eta}(n),
$$

where $\mathbf{y}_{u}(n)$ is the vector of correlation outputs corresponding to the $u$ th BS, at different time lags between 0 and maximum channel delay spread $\tau_{\max } T_{s}$. It is defined as $\mathbf{y}_{u}(n)=$ $\left[y_{u}(n, 0), \ldots, y_{u}\left(n, \tau_{\max } T_{s}\right)\right]^{T} \in \mathbb{C}^{\left(\tau_{\max }+1\right) \times 1}$. The matrix $\mathbf{G}_{u, u}$ is the pulse shape deconvolution matrix with element $g_{i, j}=$ $\sqrt{E_{b_{u}}} \Re_{u, u}(i-j)$, for $i, j=0, \ldots, \tau_{\max }, \mathbf{v}_{\eta}(n)$ is the sum of Inter-Chip-Interference (ICI), Inter symbol Interference (ISI), MAI, and AWGN noises after the despreading operation. The vector $\mathbf{h}_{u}(n)$ of elements $h_{l, u}$ is defined such that 


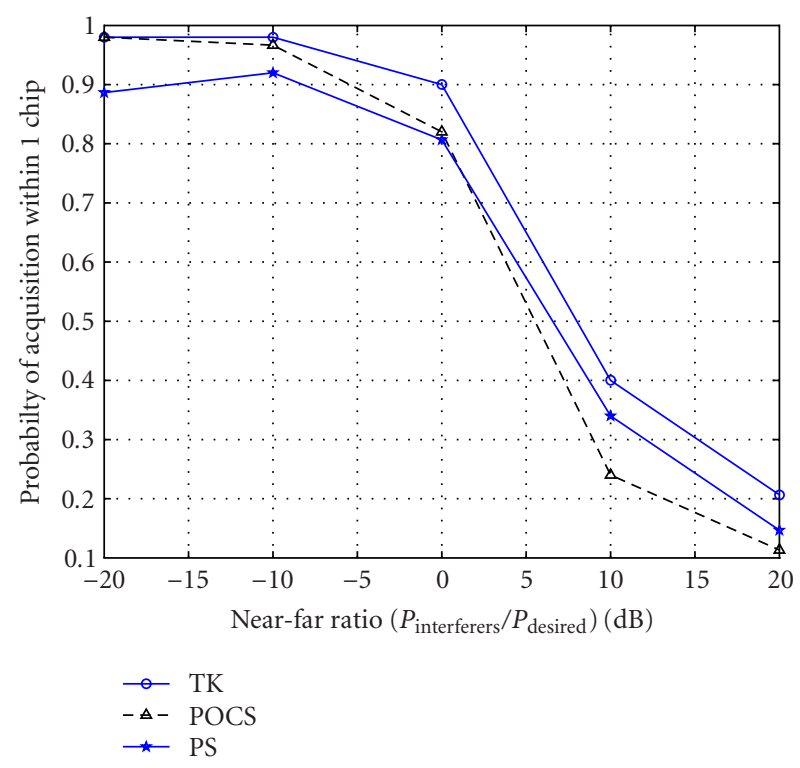

(a) Acquisition probability of first path.

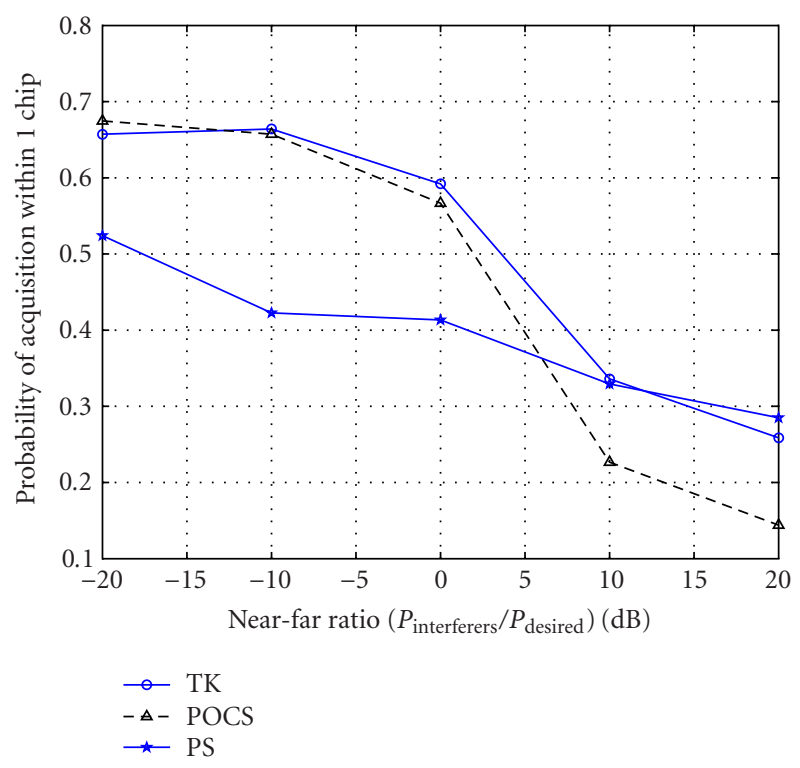

(b) Acquisition probability of all paths.

FIGURE 2: Probability of acquisition within 1 chip in closely spaced multipaths downlink WCDMA transmission using TK, POCS, and PS algorithms, $N_{\mathrm{BS}}=3, N_{u}=32, S_{F}=256, N_{s}=8, L=5$, and $E_{b} / N_{0}=10 \mathrm{~dB}$.

$h_{l, u}=0$ if no multipath is present at the time delay $l$, and $h_{l, u}=\alpha_{l, u}$ if the index $l$ corresponds to a true path location. Therefore, resolving multipath components refers to the problem of estimating the nonzero elements of the unknown gain vector $\mathbf{h}_{u}(n)$. The POCS estimation is an iterative process. The estimates at the $(k+1)$ th iteration can be written as [10]

$$
\begin{aligned}
\tilde{\mathbf{h}}_{u}(n)^{(k+1)}=\tilde{\mathbf{h}}_{u}(n)^{(k)}+ & \left(\frac{1}{\lambda_{\mathrm{POCS}}} \mathbf{I}+\mathbf{G}_{u, u}^{H} \mathbf{G}_{u, u}\right)^{-1} \\
& \times \mathbf{G}_{u, u}^{H}\left(\mathbf{y}_{u}(n)-\mathbf{G}_{u, u} \tilde{\mathbf{h}}_{u}(n)^{(k)}\right),
\end{aligned}
$$

where $\lambda_{\text {POCS }}$ is a constant determining the convergence speed and $\mathbf{I}$ is the unity matrix. The threshold used in the multipath detection is set adaptively, based on the estimation of signalto-noise ratio (SNR) in the system [29].

Figure 2 shows the probability of acquiring the first path (plot "a") and acquiring all the paths (plot "b") within 1 chip error using TK, POCS, and PS algorithms. The channel profile is Rayleigh with probability $p_{R}=0.9$ and Rician with exponential distribution Rician factor of mean $\mu_{R}=4$ with probability 0.1 . The channel has 5 paths with average powers $0,-2,0,-1$, and $-3 \mathrm{~dB}$. The acquisition probability is computed over $N_{\text {rand }}$ random realizations of the channel, $N_{\text {rand }}=150$. We see that at low Near Far Ratio (NFR) values (up to $0 \mathrm{~dB}$ ), it is possible to acquire all the paths within 1 chip in at least $60 \%$ of the cases with TK algorithm. However, the probability is much higher for the first path. This proves that the assumption of initial delay error for the EKF estimation within 1 chip is quite reasonable.

\subsection{EKF for joint estimation with closely spaced paths}

A downlink multiuser WCDMA scenario was considered with $L$ paths, the first one being either Rayleigh or Rician. The channel is supposed to be Rayleigh with probability $p_{R}$ and Rician with probability $1-p_{R}$. The delay separation between successive paths are uniformly distributed in $\left[T_{c} / N_{s} ; T_{c}\right]\left(N_{s}=8\right)$.

In Figure 3, we show the tracking trajectory of both delays and channel coefficients of the first arriving path for $L=$ 4 , with tracking delay error initialized at $N_{\text {init }}=\tau-\hat{\tau}=0.5 T_{c}$.

The matrix of the average path powers is

$$
P_{\mathrm{BS}}=\left(\begin{array}{cccc}
0 & -2 & -2 & -3 \\
-1 & -1 & -4 & -5 \\
-2 & -1 & -4 & -6 \\
-2 & -2 & -4 & -5
\end{array}\right) \mathrm{dB} .
$$

The first row corresponds to the average path powers of the desired BS. The simulation shows that EKF is able to track quite accurately the delays and the complex channel coefficients by using the IC scheme. In Figure 4, we show the probability of acquiring correctly the delay of the first arriving path within an error of 1 sample $\left(1 / N_{s}\right.$ chip) with and without IC algorithm. The channel from each BS has 3 closelyspaced paths. The corresponding average powers are

$$
P_{\mathrm{BS}}=\left(\begin{array}{ccc}
0 & -1 & -4 \\
-3 & -2 & -4 \\
-1 & -2 & -4 \\
-2 & -2 & -4
\end{array}\right) \mathrm{dB} .
$$




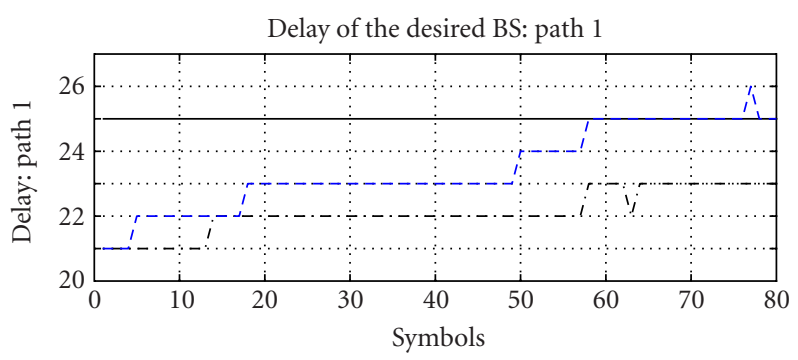

- True delay

. - . Estimated delay: no IC

- - Estimated delay: with IC

(a)

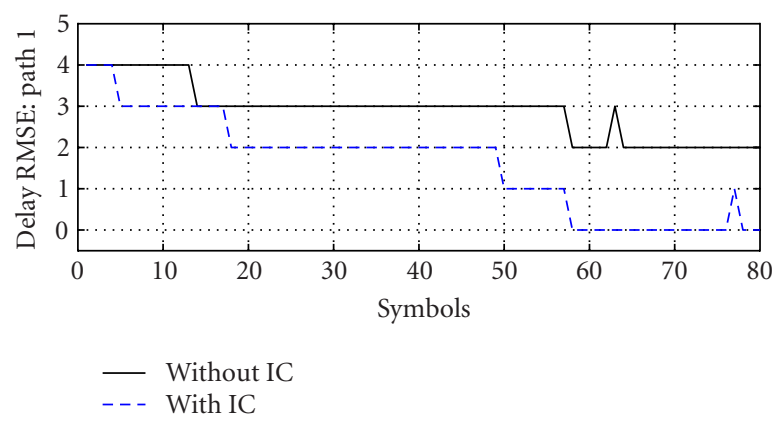

(b)

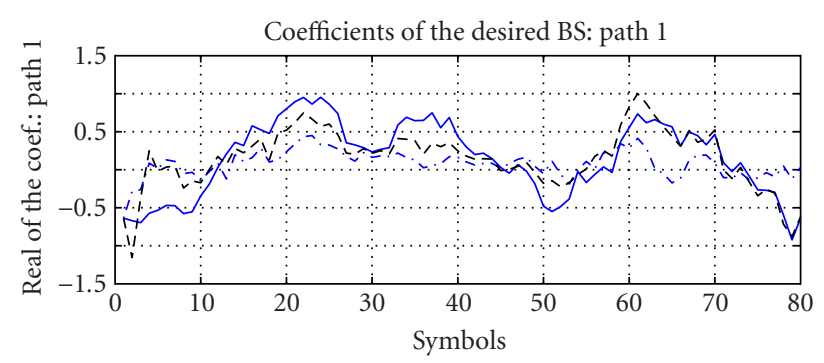

- True coefficient

- - Estimated coefficient: no IC

- - - Estimated coefficient: with IC

(c)

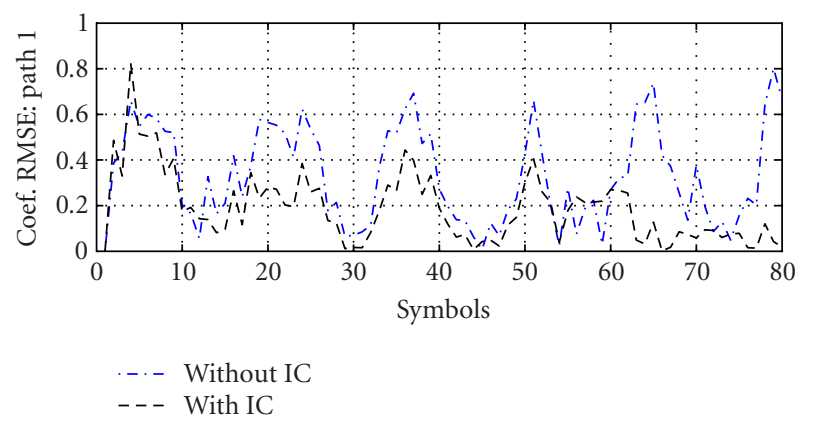

(d)

Figure 3: EKF-based desired BS estimation for four closely spaced paths, $N_{\mathrm{BS}}=4, N_{u}=8, E_{b} / N_{0}=10 \mathrm{~dB}, S_{F}=256$, and $N_{s}=8$.

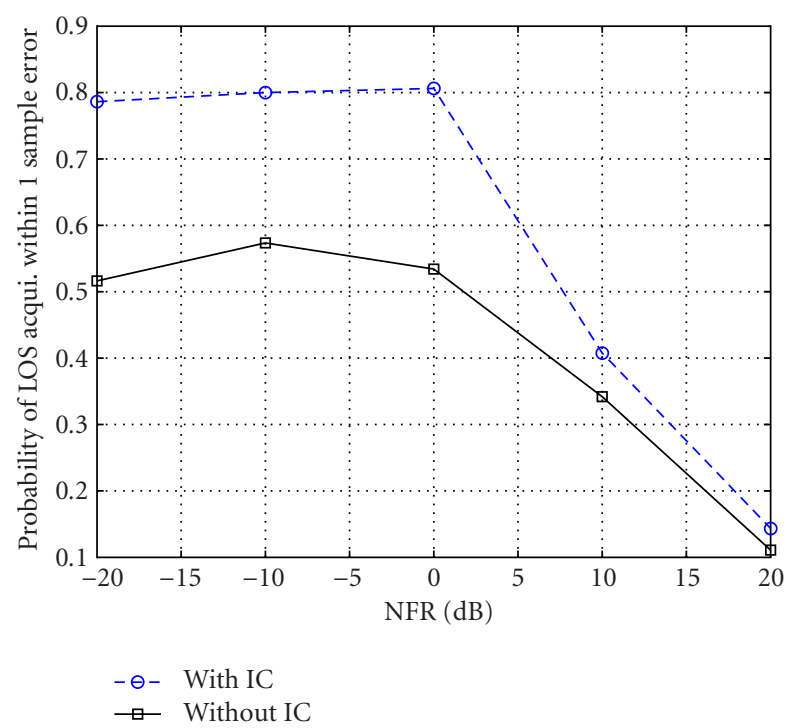

FIGURE 4: Probability of first arriving path acquisition within 1 sample error with and without IC. Three closely spaced fading channel paths, $N_{\mathrm{BS}}=4, p_{R}=0.9, E_{b} / N_{0}=8 \mathrm{~dB}, S_{F}=256, N_{s}=8, N_{u}=32$, $N_{\text {rand }}=200$.

The channel profile from the desired BS is Rayleigh with probability $p_{R}=0.9$ and it is Rician with probability 0.1 . The
Rician factor is exponentially distributed with mean $\mu_{R}=4$. The acquisition probability is computed over $N_{\text {rand }}$ random realizations of the channel, $N_{\text {rand }}=200$. We can see that it is possible to achieve $20 \%$ to $30 \%$ gain in the probability of first-arriving path acquisition by using the IC algorithm at low NFR values. The tracking of the first-arriving path can be achieved in up to $80 \%$ of the cases with IC. However, at high NFR, the feedback propagation error in EKF, when the interference is strong, prevents the correct tracking of the delay. The initial delay and covariance errors have a major effect on the convergence of the EKF, that is, bad initialization may lead to divergence of the algorithm.

\subsection{LOS detection}

First, we show the performance of PTS-based LOS detection. Then, we show the performance of Rician factor-based algorithm. We consider a relatively fast fading channel with mobile velocity $v=80 \mathrm{~km} / \mathrm{h}(22.22 \mathrm{~m} / \mathrm{s})$. In the statistical test, the decision is made on $N_{\text {slots }}$ slots basis, with $N_{\text {slots }} \in\{50,100,500,1000,1500,2000,4000\}$. Independent points spaced at $\Delta t_{\text {coh }}$ apart are taken within the decision interval. In WCDMA, 1 slot is $t_{\text {slot }}=0.6667$ milliseconds and for $S_{F}=256$, there are 10 symbols per slot. The confidence level in the decision was $99.99 \%$ [23]. Table 1 shows the comparison of the measured data distribution of the first path against several distributions: Rayleigh, Rician, Gaussian, and Lognormal. 
TABLE 1: Probabilities of accepting a certain distribution with a confidence level of $99.99 \%$. Rayleigh and Rician channels $\left(K_{r}=15.5 \mathrm{~dB}\right)$ and $v=22.22 \mathrm{~m} / \mathrm{s}$.

\begin{tabular}{rrccccc}
\hline Rayleigh channel & $N_{\text {slots }}$ & $P_{\text {Rayleigh }}$ & $P_{\text {Rician }}$ & $P_{\text {Normal }}$ & $P_{\text {Lognormal }}$ & Decision \\
\hline 50 & 0.993 & 1 & 1 & 0.83 & None \\
& 100 & 0.993 & 1 & 0.98 & 0.76 & None \\
& 500 & 1 & 1 & 0.86 & 0 & None \\
& 1000 & 1 & 1 & 0.40 & 0 & None \\
& 1500 & 1 & 1 & 0.10 & 0 & NLOS \\
\hline Rician channel & 2000 & 1 & 1 & 0 & 0 & NLOS \\
\hline & $N_{\text {slots }}$ & $P_{\text {Rayleigh }}$ & $P_{\text {Rician }}$ & $P_{\text {Normal }}$ & $P_{\text {Lognormal }}$ & Decision \\
& 50 & 1 & 1 & 1 & 0.04 & None \\
& 100 & 1 & 1 & 1 & 0 & None \\
& 500 & 1 & 1 & 0 & 0 & NLOS \\
& 1000 & 1 & 1 & 0 & 0 & NLOS \\
& 1500 & 0 & 1 & 0 & 0 & LOS \\
\hline
\end{tabular}

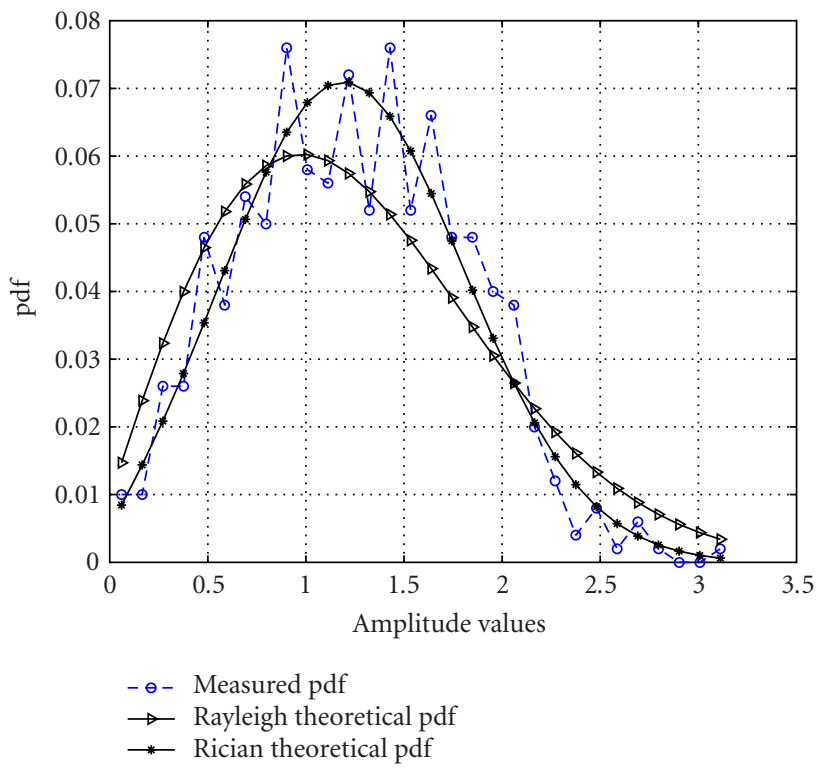

(a)

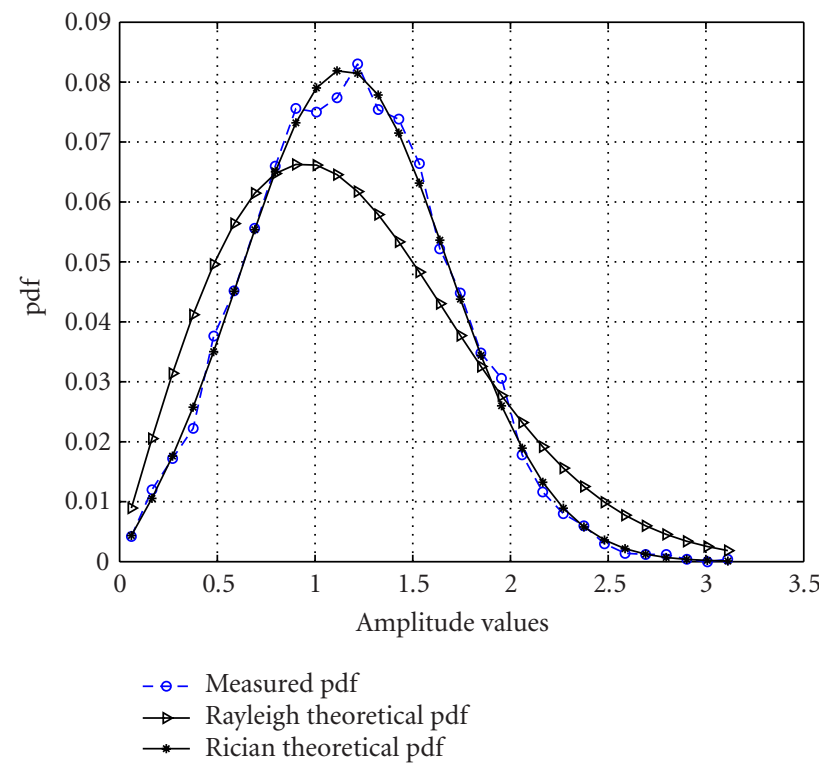

(b)

Figure 5: Estimated and theoretical Rayleigh and Rician pdfs for $N_{\text {slots }}=500$ (plot (a)) and $N_{\text {slots }}=1500$ (plot (b)). Rician channel profile $\left(K_{r}=15.5 \mathrm{~dB}\right), v=22.22 \mathrm{~m} / \mathrm{s}$.

When the channel is Rayleigh distributed (i.e., NLOS case), we see that at least 1500 slots are needed to decide Rayleigh and Rician. This is not contradictory as the Rayleigh distribution is a particular case of Rician. Hence, the overall decision will be Rayleigh and the LOS component will be absent. If we use a lower number of slots (e.g., 100 slots), the distribution cannot be established, as we also detect normal distribution with probability 0.98 , and Lognormal distribution with probability 0.76 . Also, in the case of Rician channel profile (i.e., LOS case), at least 1500 slots are needed to decide Rician distribution. We point out that for LOS case, the statistical test for Rayleigh distribution should provide
$P_{\text {Rayleigh }}=0$. In this case, the number of independent points needed for the decision is $N=880$, which is obtained from

$$
N=t_{\text {slot }} \frac{N_{\text {slots }}}{\Delta t_{\text {coh }}} .
$$

In Figure 5, we show the similarities between estimated pdf, theoretical Rayleigh, and theoretical Rician pdfs when the channel is Rician with $N_{\text {slots }}=500$ and $N_{\text {slots }}=1500$. We can see that the measured data curve and Rician curve have good fitting for the later case. This technique can be used efficiently in continuous time measurement mode when the mobile can keep track of the channel estimates over several 


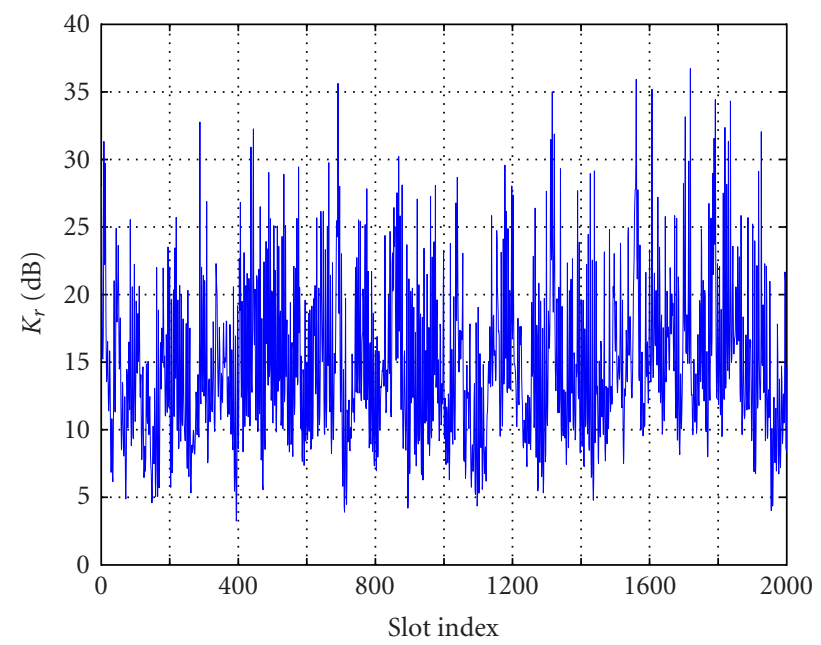

(a)

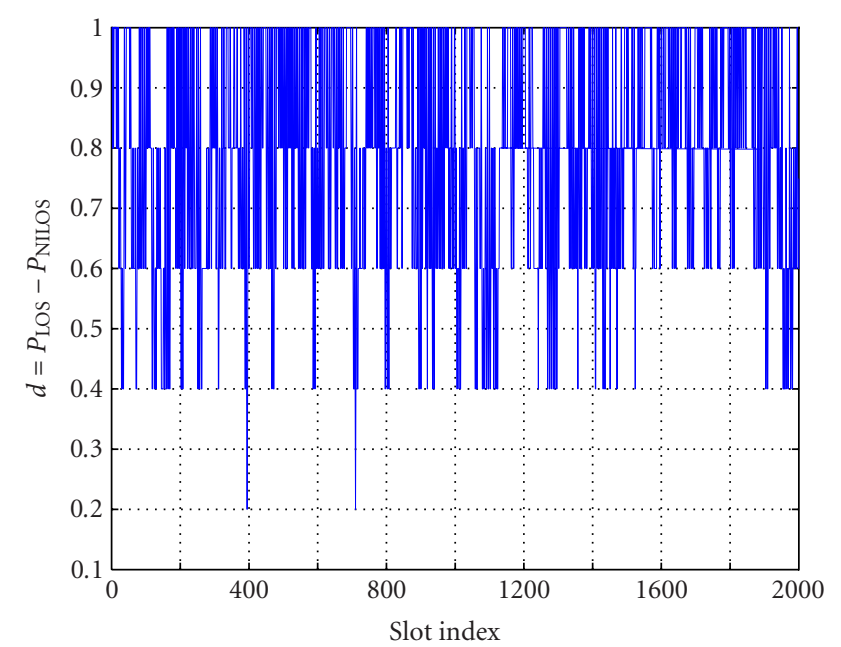

(b)

Figure 6: Estimated Rician factor $K_{r}$ (plot (a)) and the probability distance $d$ (plot (b)). Channel profile Rician with $K_{r}=15.5 \mathrm{~dB}$ and $v=22.22 \mathrm{~m} / \mathrm{s}$.

TABle 2: Probabilities of accepting a certain distribution using Rician factor-based algorithm. Rayleigh and Rician channel $\left(K_{r}=\right.$ $15 \mathrm{~dB}$ ) and $v=22.22 \mathrm{~m} / \mathrm{s}$.

\begin{tabular}{rrrccc}
\hline Rayleigh channel & $N_{\text {slots }}$ & $p_{\text {NLOS }}$ & $p_{\text {LOS }}$ & $d_{\text {mean }}$ & Decision \\
\hline & 1 & 0.0917 & 0.9083 & 0.8165 & LOS \\
10 & 0.5760 & 0.4240 & -0.1520 & NLOS \\
50 & 0.8133 & 0.1867 & -0.6267 & NLOS \\
& 100 & 0.9000 & 0.1000 & -0.8000 & NLOS \\
\hline Rician channel & $N_{\text {slots }}$ & $p_{\text {NLOS }}$ & $p_{\text {LOS }}$ & $d_{\text {mean }}$ & Decision \\
\hline & 1 & 0.0975 & 0.9026 & 0.8 & LOS \\
10 & 0.0272 & 0.9728 & 0.94 & LOS \\
50 & 0.0433 & 0.9567 & 0.91 & LOS \\
& 100 & 0.0918 & 0.9082 & 0.8164 & LOS \\
500 & 0 & 1 & 1 & LOS \\
\hline
\end{tabular}

milliseconds, for example, the $\mathrm{CPICH}$ signal coming from the serving BS.

By applying Algorithm 1 to the same channel profiles tested with the pdf-based technique, we can obtain faster decision on whether the channel is Rayleigh or Rician. The minimum and maximum edges $B_{\min }$ and $B_{\max }$ are -20 and $+20 \mathrm{~dB}$, respectively, and the number of subintervals considered is $(M+1)=10$. Figure 6 shows the estimated Rician factor in $\mathrm{dB}$ (plot (a)) and the distance $d=P_{\mathrm{LOS}}-P_{\mathrm{NLOS}}$ (plot (b)) when the Rician factor is computed on a slot by slot basis. Table 2 shows the means $p_{\mathrm{LOS}}$ and $p_{\mathrm{NLOS}}$, respectively, of the probabilities $P_{\mathrm{LOS}}$ and $P_{\mathrm{NLOS}}$, the mean distance $d_{\text {mean }}$ of $d$, and the corresponding decision when the Rician factor is computed over $N_{\text {slots }} \in\{1,10,50,100,500\}$ slots.

For the case of Rayleigh channel, we see that the decision based on 1 slot is not possible, the estimated Rician factor in this case is too high, and the decision will be Rician. At least 10 slots are needed to decide safely that the distribution is Rayleigh. However, in the case of Rician channel, it is quite easy to decide the presence of LOS even on a slot-by-slot basis. To show the performance of Rician factor-based algorithm, we considered a channel with succession of Rayleigh and Rician fading. The estimation of the Rician factor is done on a frame-by-frame basis ( 1 frame $=15$ slots). Figure 7 shows that the true Rician factor versus the estimated Rician factor in $\mathrm{dB}$ (plot (a)) and the distance $d=P_{\mathrm{LOS}}-P_{\mathrm{NLOS}}$ (plot (b)). During the first 200 frames and between frames of index 500 and 600, the channel is Rayleigh $\left(K_{r}[\mathrm{~dB}]=\infty\right)$. The minimum and maximum edges $B_{\min }$ and $B_{\max }$ are -20 and $+20 \mathrm{~dB}$, respectively, and the number of subintervals is $(M+1)=10$. We point out that these two edges, $B_{\min }$ and $B_{\max }$, should be set adaptively, based on the noise level in the system. It is clear that during the first 400 frames, $d_{\text {mean }}<0$, where $d_{\text {mean }}=\operatorname{mean}\left\{d_{i}, 0 \leq i \leq 400\right\}$, which indicates the absence of LOS component, even if we have Rician distribution during 200 frames. This is due to the fact that for $K_{r}=-6 \mathrm{~dB}$, which is very low, the Rician distribution is very similar to Rayleigh. However, when the Rician factor is $6,15.5$, or $20 \mathrm{~dB}$, it is quite easy to decide the presence of LOS component.

The two presented techniques for LOS detection are making a trade-off between short observation time and noiselevel estimation. The first technique that is based on pdf estimation does not need any estimation of the noise level, but it requires long observation time, which is not a limitation in continuous time measurement. The second technique which uses much lower observation time needs an estimate of the noise level to set adaptively the thresholds $B_{\min }$ and $B_{\max }$.

\section{CONCLUSIONS}

New techniques of LOS/NLOS detection for mobile positioning for WCDMA system have been presented, based on EKF estimation and statistic tests-based decisions. The delays and channel coefficients are jointly estimated using EKF 


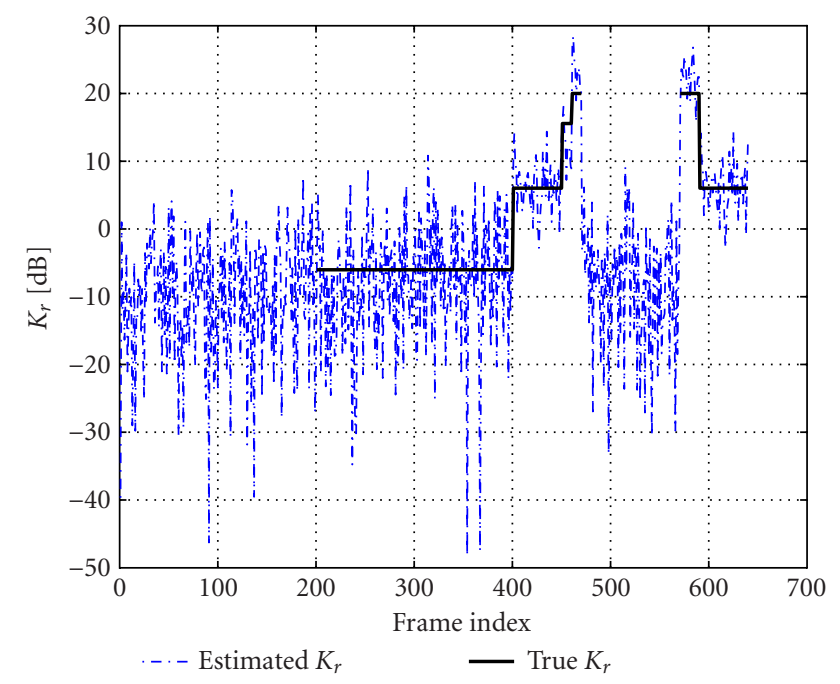

(a)

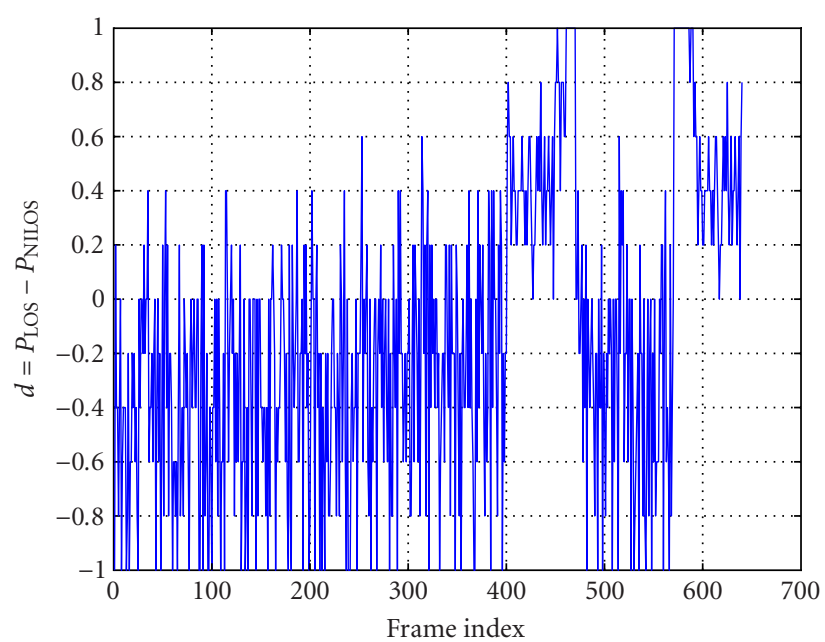

(b)

FIGURE 7: Estimated Rician factor $K_{r}$ (plot (a)) and the probability distance $d$ (plot (b)). Channel profile: combined Rayleigh-Rician and $v=22.22 \mathrm{~m} / \mathrm{s}$.

with an IC scheme in the context of closely spaced paths in multicell WCDMA transmission. The simulation results showed that the tracking of the first-arriving path can be achieved efficiently with a probability of acquisition varying from $40 \%$ to $80 \%$ of the cases in good NFR conditions (NFR $\leq 10 \mathrm{~dB}$ ). The channel coefficient estimates are then used for LOS/NLOS detection. We have presented two statistics-based techniques. The first one is using curve fitting criteria. This method requires the storage of $N$ independent points in the mobile terminal updated at least at coherence time interval $\left(\Delta t_{\mathrm{coh}}\right)$ (about 880 points). We showed that this technique can provide quite satisfactory decision on whether the LOS component is present or not. The second technique is based on the estimation of Rician factor and can be used when the measurement interval is constrained in time. We found that in moderate-to-high mobility case, one frame is enough to carry reliable decision on whether the LOS component is present or not. However, the decision parameters should be updated according to the noise level for best performance.

\section{ACKNOWLEDGMENTS}

This research was supported by Nokia, Nokia Foundation, and by the Graduate School in Electronics, Telecommunications, and Automation (GETA).

\section{REFERENCES}

[1] FCC docket No. 94-102, Fourth Memorandum Opinion and Order, September 2000.

[2] J. Reed, K. Krizman, B. Woerner, and T. Rappaport, "An overview of the challenges and progress in meeting the e911 requirement for location service," IEEE Communications Magazine, vol. 36, no. 4, pp. 30-37, 1998.
[3] R. E. Játiva and J. Vidal, "GLRT detector for NLOS error reduction in wireless positioning systems," in Proc. IST Mobile and Wireless Telecommunications Summit, Thessaloniki, Greece, June 2002.

[4] N. Yousef and A. Sayed, "Detection of fading overlapping multipath components for mobile-positioning systems," in Proc. IEEE International Conference on Communications (ICC '01), vol. 10, pp. 3102-3106, Helsinki, Finland, June 2001.

[5] M. Wylie and J. Holtzman, "The non-line of sight problem in mobile location estimation," in Proc. 5th IEEE International Conference on Universal Personal Communications (ICUPC '96), vol. 2, pp. 827-831, Cambridge, Mass, USA, September-October 1996.

[6] M. Wylie and J. Holtzman, "Method and system for mobile location estimation," US Patent Application Publication, US005974329A, Rutgers University, NJ, USA, October 1999.

[7] P. Chen, "Mobile position location estimation in cellular systems," Tech. Rep. WINLAB-TR-181, Rutgers University, Piscataway, NJ, USA, 1999.

[8] T. Ostman, S. Parkvall, and B. Ottersten, "Improved MUSIC algorithm for estimation of time delays in asynchronous DS-CDMA systems," in Proc. Asilomar Conference of Signals, Systems and Computers (ASILOMAR '97), vol. 1, pp. 838-842, Pacific Grove, Calif, USA, November 1997.

[9] Z. Kostic and G. Pavlovic, "Resolving sub-chip spaced multipath components in CDMA communication systems," in Proc. 43rd IEEE Vehicular Technology Conference (VTC '93), vol. 1, pp. 469-472, Secaucus, NJ, USA, May 1993.

[10] D. Colclough and E. Titlebaum, "Delay-doppler POCS for specular multipath," in Proc. IEEE Int. Conf. Acoustics, Speech, Signal Processing (ICASSP '02), vol. 4, pp. 3940-3943, Orlando, Fla, USA, May 2002.

[11] R. Iltis, "Joint estimation of PN code delay and multipath using the extended Kalman filter," IEEE Trans. Communications, vol. 38, no. 10, pp. $1677-1685,1990$.

[12] J. K. Kyeong and R. Iltis, "Joint detection and channel estimation algorithms for QS-CDMA signals over time-varying channels," IEEE Trans. Communications, vol. 50, no. 5, pp. 845-855, 2002. 
[13] S. Glisic and B. Vucetic, Spread Spectrum CDMA Systems for Wireless Communications, Artech House Publishers, Norwood, Mass, USA, 1997.

[14] 3GPP, "Physical layer-general description," Tech. Rep. TS 25.201 V3.0.0, 3rd Generation Partnership Project, Sophia Antipolis, France, 1999, available at http://www.3gpp.org/ftp/ specs/latest/R1999.

[15] B. D. O. Anderson and J. B. Moore, Optimal Filtering, Prentice-Hall, Englewood Cliffs, NJ, USA, 1979.

[16] C. Komninakis, C. Fragouli, A. Sayed, and R. Wesel, "Channel estimation and equalization in fading," in Proc. 33rd Asilomar Conference of Signals, Systems and Computers, vol. 2, pp. 11591163, Pacific Grove, Calif, USA, November 1999.

[17] T. Lim and L. Rasmussen, "Adaptive symbol and parameter estimation in asynchronous multiuser CDMA detectors," IEEE Trans. Communications, vol. 45, no. 2, pp. 213-220, 1997.

[18] P. Diaz and R. Agusti, "Design and implementation of an EKF-based tracking synchronism scheme for an indoor DS/CDMA system," in Proc. 46th IEEE Vehicular Technology Conference (VTC '96), vol. 1, pp. 601-605, Atlanta, Ga, USA, April 1996.

[19] M. Simon and M. Alouini, Digital Communications over Fading Channels: a Unified Approach to Performance Analysis, John Wiley \& Sons, NY, USA, 2000.

[20] T. Rappaport, Wireless Communications: Principles and Practice, Prentice-Hall, Englewood Cliffs, NJ, USA, 1996.

[21] M. Cedervall, "Mobile positioning for third generation WCDMA systems," in Proc. IEEE International Conference on Universal Personal Communications (ICUPC '98), vol. 2, pp. 1373-1377, Florence, Italy, October 1998.

[22] Y. Jeong, H. You, W. Lee, D. Hong, and D. Youn, "A wireless position location system using forward pilot signal," in Proc. IEEE Vehicular Technology Conference (VTC '00), vol. 2, pp. 1354-1357, Tokyo, Japan, May 2000.

[23] A. Papoulis, Probability, Random Variables, and Stochastic Process, McGraw-Hill, NY, USA, 3rd edition, 1991.

[24] R. Hamila, E. Lohan, and M. Renfors, "Subchip multipath delay estimation for downlink WCDMA system based on Teager-Kaiser operator," IEEE Communications Letters, vol. 7, no. 1, pp. 1-3, 2003.

[25] Z. Kostic and G. Pavlovic, "CDMA RAKE receiver with subchip resolution," US Patent Publication, US5648983, Lucent Technologies, Murray Hill, NJ, USA, July 1997.

[26] E. Sourour, G. Bottomley, and R. Ramesh, "Delay tracking for direct sequence spread spectrum systems in multipath fading channels," in Proc. IEEE Vehicular Technology Conference (VTC'99), vol. 1, pp. 422-426, Houston, Tex, USA, May 1999.

[27] E. Lohan, R. Hamila, and M. Renfors, "Performance analysis of an efficient multipath delay estimation approach in a CDMA multiuser environment," in Proc. 12th IEEE International Symposium on Personal, Indoor, and Mobile Radio Communications (PIMRC '01), pp. 6-10, San Diego, Calif, USA, September-October 2001.

[28] R. Hamila, Synchronization and multipath delay estimation algorithms for digital receivers, Ph.D. thesis, Tampere University of Technology, Tampere, Finland, 2002.

[29] E. Lohan, A. Lakhzouri, and M. Renfors, "LOS estimation in overlapped multipath WCDMA scenarios via adaptive threshold," in Proc. IEEE Workshop on Signal Processing Advances in Wireless Communications (SPAWC '03), Rome, Italy, June 2003.
Abdelmonaem Lakhzouri was born in Tunis, Tunisia, in 1975. He received the M.S. degree in signal processing from the Ecole Supérieure des communications de Tunis, Tunisia, in 1999, and the Diplôme d'Etudes Approfondies (DEA) degree in communications from Ecole Nationale d'ingénieurs de Tunis, Tunisia, in 2001. From 2000 till now, he has been a Researcher at the Institute of Communication Engineering at

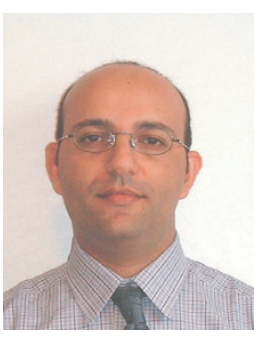
Tampere University of Technology, Finland, where he is working toward the Ph.D. degree in mobile communications. His research topics include multipath delay estimation for mobile positioning applications, CDMA receiver design, and programmable implementation of flexible receivers.

Elena Simona Lohan received the M.S. degree in electrical engineering from the Politehnica University of Bucharest, Romania, in 1997, and the Diplôme d'Etudes Approfondie (DEA) degree in econometrics from Ecole Polytechnique, Paris, France, in 1998. From 1998 till now, she has been a Researcher at the Institute of Communications Engineering at Tampere University of Technology, Finland. Her research interests include CDMA receiver design, fading channel estimation, and mobile phone positioning technologies.

Ridha Hamila received the M.S., Lic.Tech., and Ph.D. degrees from the Department of Information Technology, Tampere University of Technology (TUT), Tampere, Finland, in 1996, 1999, and 2002, respectively. From 1995 to 1996, he was a Research Assistant at the Signal Processing Laboratory, TUT. In 1997, he joined the Telecommunications Laboratory at TUT where he was a Researcher and Lecturer. From 2002 to

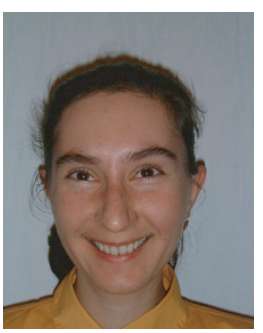
2003, he was with Nokia Networks, Espoo-Helsinki, Finland. Since 2003, he has been with Etisalat College of Engineering, UAE, as an Assistant Professor. His research interests include synchronization and multipath delay estimation for digital receivers as well as mobile phone positioning technologies and software defined radios.

Markku Renfors was born in Suoniemi, Finland, in 1953. He received the Diploma Engineer, Licentiate of Technology, and Doctor of Technology degrees from Tampere University of Technology (TUT), Tampere, Finland, in 1978, 1981, and 1982, respectively. From 1976 to 1988 , he held various research and teaching positions at TUT. From 1988 to 1991, he was a Design Manager at the Nokia Research Center and

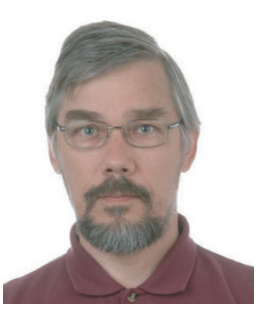
Nokia Consumer Electronics, Tampere, Finland, where he focused on video signal processing. Since 1992, he has been a Professor of telecommunications at TUT. His main research area is signal processing algorithms for flexible radio receivers and transmitters. 